\title{
Is Iron-Chelation Therapy Useful in Persons with Myelodysplastic Syndrome?
}

\author{
Chaim Hershko ${ }^{1, *}$, Robert Peter Gale ${ }^{2}$ \\ ${ }^{1}$ Department of Hematology, Shaare Zedek Medical Center, Jerusalem, Israel; ${ }^{2}$ Haematology Section, Division of Experimental \\ Medicine, Department of Medicine, Imperial College, London, UK. \\ Email: "hershkoc@netvision.net.il
}

Received July 28 ${ }^{\text {th }}, 2011$; revised August $29^{\text {th }}, 2011$; accepted September $6^{\text {th }}, 2011$.

\begin{abstract}
Iron overload in myelodysplastic syndrome (MDS) results from multiple RBC-transfusions and inappropriate increased iron absoption associated with ineffective erythropoiesis. Data from hereditary iron-loading anemias indicate longterm consequences of iron toxicity are preventable and potentially reversible by effective iron-chelation therapy (ICT). There is increasing interest in using ICT in persons with MDS because of the recent introduction of orally effective iron-chelators which are suitable for older persons. Ideally, opinions supporting the benefit of ICT in MDS should be evidenced-based, especially data from randomized trials showing better survival and, in exceptional cases, improved heart function. Such data are lacking. Nevertheless, it is possible to rely on the extensive data from trials of ICT in persons with thalassemia and to use well-defined predictors of increased risks of life-threatening complications to identify persons with MDS most likely to benefit from ICT.
\end{abstract}

Keywords: Myelodysplastic Syndrome, MDS, Iron-Chelation, NTBI, Deferoxamine, Deferiprone, Deferasirox, Transfusion Siderosis

\section{Selection of Persons with MDS for Iron-Chelating Therapy}

The choice of persons with MDS who may benefit from ICT is of continued interest and controversy [1-5]. Despite some differences, principles underlying recommendations are remarkably similar for persons with MDS and those with thalassemia. First, it is recognized that life-threatening RBC-transfusion-induced iron overload is only relevant to persons with MDS with a favorable prognosis. Second, magnitude and rate of iron-accumulation are critical. Substantial heart iron-accumulation is expected only after about 100 units (U) of RBCs [6-11; Table 1]. A person receiving 2 - 4 U RBCs monthly will only be at risk after about 2 - 4 years. Thus, recommenddations to start ICT after $20 \mathrm{U}$ RBCs are too liberal and merits consideration only if the rate of RBC-transfusions predicts $\geq 100 \mathrm{U}$ RBCs in $\leq 4$ years. Lastly, because serum ferritin in persons with MDS is affected by inflammation, infection and leukemia burden, it is an unreliable indicator of iron-overload $[12,13]$. Hence, currently proposed threshold ferritin levels of 1000 - $2500 \mathrm{ng} / \mathrm{dL}$ should be supplemented or replaced by estimates of ironoverload based on state-of-the-art MRI methodology which directly assesses tissue iron-accumulation. When such technology is unavailable, the RBC-transfusion threshold of $\geqslant 100 \mathrm{U}$ is a practical way to identify persons with MDS at high-risk of life-threatening iron-accumulation.

The importance of ICT in MDS is not universally accepted [14]. Although favorable effects of ICT in thalassemia include reversal of arrhythmias, improvement in left ventricular ejection-fraction, arrest of hepatic fibrosis and decreased glucose tolerance are well-documented, it is uncertain whether these outcomes apply to persons with MDS receiving ICT.

\section{Choice of Iron-Chelators in Persons with MDS}

A comparison of the 3 leading chelators is presented in Table 2.

Deferoxamine: Because of its proved efficacy and the extensive experience in persons with thalassemia, deferoxamine (DFO) is considered the gold-standard for ICT [15]. However, compliance with the rigorous requirements of daily subcutaneous DFO infusions is a serious limiting-factor. In practice, elderly persons with MDS are not reasonable candidates to receive DFO. 
Table 1. Relation of heart iron-accumulation to RBC-transfusion volume in transfused adults without thalassemia.

Buja et al. (1971) Diverse diseases. Clinically-important heart iron accumulation in $60 \%$ of subjects after $101-200$ and $100 \%$ of subjects after 201 - 300 U RBCs [6];

Schafer et al. (1981) Anemias. After 60 - 210 U RBCs left ventricular function was impaired in only the most heavily RBC-transfused subjects [7];

Jensen et al. (2003) Diverse diseases. Abnormal heart iron-accumulation in subjects with liver iron concentration $>22.3 \mathrm{mg} / \mathrm{g}$ dry weight equal to $89 \mathrm{U} \mathrm{RBCs} *[8]$

DiTucci et al. (2008) Anemias. Heart T2* correlated with RBC-transfusions. No subject receiving $<290 \mathrm{~mL} / \mathrm{Kg}$ of PRBCs (equal to $101 \mathrm{U}$ RBCs) had an abnormal T2* value $(<20 \mathrm{~ms})[9]$;

Chacko J. et al. (2007) MDS. Median RBC-transfusions of 63 and $112 \mathrm{U}$ in persons not receiving or receiving ICT T2* was normal (90\%) or borderline-normal $(10 \%)[10]$;

Konen E. et al. (2007) MDS. Median RBC-transfusions of 90 U RBCs. All subjects had normal T2 [11].

*Calculated by the Angelucci formula [26] assuming a mean body weight of $75 \mathrm{~kg}$ each U RBC representing $200 \mathrm{mg}$ iron.

Table 2. Comparison of iron-chelating drugs [15].

\begin{tabular}{lccc}
\hline & Deferoxamine & Deferiprone & Deferasirox \\
\hline MW (Da) & 657 & 139 & 373 \\
Chelating property & Hexadentate & Bidentate & Tridentate \\
Iron-binding affinity (pM) & 26.6 & 19.9 & 22.5 \\
Chelating efficiency (\%) & 13 & 7 & 27 \\
Dose mg/kg/d & $30-50$ & $75-100$ & Oral, once daily \\
Route & s.c. or i.v. 8 - 12 times daily & $12-16 \mathrm{~h}$ \\
Half-life & $20-30$ min, 5 days/week & $3-4$ h & High \\
Lipid solubility & Low & Intermediate & Fecal \\
Excretion & Urine/fecal & Urine & 80 \\
Peak plasma levels (pM) & 5 - 10 & $90-450$ & GI, rash, ocular, auditory, kidney \\
Adverse effects & Ocular, auditory growth, local reactions, allergy & GI, arthralgia, $\downarrow$ PMN
\end{tabular}

Deferiprone: Because of its smaller size and higher lipophilicity compared with DFO, deferiprone (DFP) readily enters cells and may access intracellular chelatable iron more effectively than DFO. The cardio-protective effect of DFP is the most remarkable advantage of this compound. Weekly WBCs are recommended because of the risk of drug-induced agranulocytosis. This complicates the management of ICT in persons with MDS with disease or treatment-related granulocytopenia.

Deferasirox: DFS is a synthetic iron-chelator with a plasma half-life of 11-19 hours which allows once-daily oral dosing. DFS is highly-efficient in removing nontransferrin bound iron from the blood [16] and is usually well-tolerated. and is particularly suitable in older persons with MDS. It is premature to conclude whether DFS improves survival of persons with MDS. Recommended monitoring includes monthly creatinine and liver function tests.

Combined Iron-Chelating Drugs: Persons failing single-drug ICT may respond to a combination of ironchelators. There are considerable data on combined drugs in persons with thalassemia. However, there are insufficient data to recommend this strategy in persons with MDS.

\section{Impact of ICT on Survival in MDS}

There are few uncontrolled studies reporting the impact of ICT on survival of persons with MDS. Retrospective analyses of small studies of persons with MDS receiving or not receiving ICT suggest better survival amongst the former $[17,18]$. The most recent and extensive non-randomized study [19] included 53 subjects receiving ICT 
Table 3. Controversies regarding ICT in MDS.

\begin{tabular}{ll}
\hline \multicolumn{1}{c}{ Claim } & \multicolumn{1}{c}{ Ambiguity } \\
\hline ICT should be started at serum ferritins $\geq 1000-2500 \mathrm{ng} / \mathrm{dL}$. & Feritin is an acute phase protein and is unreliable in MDS [12,13]. \\
ICT is indicated when RBC-transfusions $\geq 20 \mathrm{U}$. & $\begin{array}{l}\text { Clinically-important heart iron-accumulation occurs only after } \geq \\
\text { RBC-transfusions } \geq 100 \mathrm{U} \text { RBCs in } \leq 2-4 \text { years [6-11]. }\end{array}$ \\
$\begin{array}{l}\text { ICT improves survival and prevents leukemia } \\
\text { transformation. }\end{array}$ & $\begin{array}{l}\text { Non-randomized trial data which may reflect selection biases } \\
{[17-19] .}\end{array}$ \\
$\begin{array}{l}\text { Prospective controlled tials of ICT in MDS are } \\
\text { needed. }\end{array}$ & $\begin{array}{l}\text { Low compliance and high-rates of competing causes } \\
\text { of morbidity and death in older persons may obscure benefits. }\end{array}$ \\
\hline
\end{tabular}

and 44 controls. Median survivals were 124 and 53 months suggesting a benefit for ICT. However, several potential selection biases and issues complicate this analysis: 1) causes of death did not differ between the cohorts; 2) end-of study serum ferritin levels were similar; 3) persons not receiving ICT were older and had somewhat worse prognoses; and 4) a higher rate of progression to AML in the no ICT cohort could reflect a worse prognosis independent of ICT.

The major limitation of retrospective and non-randomized survival studies is subject selection biases. Persons expected to live long enough to benefit from ICT are more likely to receive ICT biasing the survival analysis. Although the need for randomized studies is widely accepted, it is unlikely these will resolve controversy over using ICT in MDS. The high rate of competing causes of morbidity and death in older persons with MDS may obscure a possible benefit of ICT [19]. Consequently, long-term use of ICT will likely rest on thoughtful initial evaluation of each person considering relevant clinical data including: 1) likelihood of sufficiently long survival to receive $\geq 100 \mathrm{U}$ RBCs within $\leq 2$ - 4 years; and (2) periodic evaluation of the severity and distribution of iron-accumulation using state-of-the-art MRI techniques. Current claims and ambiguities regarding ICT in MDS are summarized in Table 3.

\section{Effects of ICT on Hematopoiesis}

Improvement in RBC-production in MDS following ICT was first reported by Jensen et al [20] and confirmed in several subsequent reports in persons with MDS or MPN-associated myelofibrosis [21]. This effect is unpredictable and seen with several iron-chelators.

The mechanism responsible for the effect of iron chelation on hemoglobin production is unknown. In a person with homozygous glutaredoxin-5 (GRLX5) mutation with sideroblast-like microcytic anemia and iron-overload, deferoxamine treatment resulted in improved heme synthesis and the partial correction of anemia [22]. Here, the likely mechanism of improved hemoglobin synthesis could be redistribution of mitochondrial iron into the cytosol following ICT reversing repression of ALAsynthase-2 translation. This effect may be expected in only occasional persons with MDS and excessive mitochondrial iron deposition. Understanding the underlying mechanism of this effect may have practical implications.

\section{Other Benefits of ICT}

A recent review suggested removal of NTBI by ICT may protect against infection, leukemia transformation and improve transplant outcomes [23]. These claims should be interpreted cautiously. As discussed, lower risks of leukemia transformation may reflect selection-biases [19]. Also, as discussed, increased pretransplant ferritin levels may reflect co-morbidities adversely affecting transplant outcome [24] unrelated to iron-overload. Using ICT to prevent transplant-related infections is particularly hazardous because iron chelators inhibit ribonucleotide reductase and may delay or prevent engraftment [25]. Because of these considerations, we consider trials of ICT in unselected persons with MDS ill-advised [23].

\section{Conclusions}

Development of oral effective iron-chelators suitable for use in older persons has led to increasing interest in ICT in persons with MDS. Appropriate selection of persons with MDS who may benefit from ICT involves several considerations: 1) RBC-tranfusion-related iron-overload is only relevant to persons with a favourable prognosis; 2) clinically-important heart iron-accumulation is expected only after about $\geqslant 100 \mathrm{U}$ RBCs given over $\leqslant 2-4$ years; and 3 ) serum ferritin is a poor indicator of iron-overload and should be replaced by state-of-the-art MRI techniques. Retrospective analyses of non-randomized and/or uncontrolled trials of ICT in persons with MDS suggest ICT results in a decrease in iron-accumulation-related mortality and, sometimes, decreased RBC-transfusion rates. However, important selection biases are likely to operate in these studies. Randomized trials are needed to determine if there is a survival benefit correlated with ICT in persons with MDS. 


\section{REFERENCES}

[1] N. Gattermann, "The Treatment of Secondary Hemochromatosis," Deutsch Arzteblatt International, Vol. 106, No. 30, 2009, pp. 499-504. doi: 10.3238/arztebl.2009.0499

[2] G. Metzgeroth, D. Dinter, B. Schultheis, et al., "Deferasirox in MDS Patients with Transfusion-Caused Iron Overload-A Phase-II Study," Annals Hematology, Vol. 88, No. 4, 2009, pp. 301-310. doi:10.1007/s00277-008-0588-3

[3] P. Fenaux and C. Rose, "Impact of Iron Overload in Myelodysplastic Syndromes," Blood Reviews, Vol.23 Suppl. 1, 2009, pp. S15-S19. doi:10.1016/S0268-960X(09)70005-0

[4] L. Malcovati, "Red Blood Cell Transfusion Therapy and Iron Chelation in Patients with Myelodysplastic Syndromes," Clinical Lymphoma Myeloma \& Leukemia, Vol. 9, Suppl. 3, 2009, pp. S305-S311.

[5] J. M. Bennett, "MDS Foundation's Working Group on Transfusional Iron Overload. Consensus Statement on Iron Overload in Myelodysplastic Syndromes," American Journal Hematology, Vol. 83, No.11, 2008, pp. 858-861. doi:10.1002/ajh.21269

[6] L. M. Buja, W. C. Roberts, "Iron in the Heart. Etiology and Clinical Significance," American Journal Medicine, Vol. 51, No. 2, 1971, pp. 209-221. doi:10.1016/0002-9343(71)90240-3

[7] A. I. Schafer, R. G. Cheron, R. Dluhy, et al., "Clinical Consequences of Acquired Transfusional Iron Overload in Adults, The New England Journal Medicine, Vol. 304, No. 6, 1981, pp. 319-324. doi:10.1056/NEJM198102053040603

[8] P. D. Jensen, F. T. Jensen, T Christensen, et al., "Evaluation of Myocardial Iron by Magnetic Resonance Imaging during Iron Chelation Therapy with Deferrioxamine: Indication of Close Relation between Myocardial iron Content and Chelatable Iron Pool," Blood, Vol. 101, No. 11, 2003, pp. 4632-4639.

[9] A. A. Di Tucci, G. Matta, S. Deplano, et al., "Myocardial Iron Overload Assessment by T2* Magnetic Resonance Imaging in Adult Transfusion Dependent Patients with Acquired Anemias," Haematologica, Vol. 93, No. 9, 2008, pp. 1385-1388.

[10] J. Chacko, D. J. Pennell, M. A. Tanner, et al., "Myocardial Iron Loading by Magnetic Resonance Imaging T2* in Good Prognostic Myelodysplastic Syndrome Patients on Long-Term Blood Transfusions," British Journal Haematology, Vol. 138, No. 5, 2007, pp. 587-593. doi:10.1111/j.1365-2141.2007.06695.x

[11] E. Konen, H. Ghoti, O. Goitein, et al., "No Evidence for Myocardial Iron Overload in Multitransfused Patients with Myelodysplastic Syndrome Using Cardiac Magnetic Resonance T2 Technique," American Journal Hematology, Vol. 82, No. 11, 2007, pp. 1013-1016. doi:10.1002/ajh.20980

[12] Y. Matzner, A. M. Konijn and C. Hershko, "Serum Ferritin in Hematologic Malignancies," American Journal
Hematology, Vol. 9, No. 1, 1980, pp. 13-22. doi:10.1002/ajh.2830090103

[13] M. H. Lee and R. T. Means Jr, "Extremely Elevated Serum Ferritin Levels in a University Hospital: Associated Diseases and Clinical Significance," American Journal Medicine, Vol. 98, No. 6, 1995, pp. 566-571. doi:10.1016/S0002-9343(99)80015-1

[14] A. Tefferi and J. W. Vardiman, "Myelodysplastic Syndromes," The New England Journal Medicine, Vol. 361, No. 19, 2009, pp. 1872-1885. doi:10.1056/NEJMra0902908

[15] C. Hershko, "Oral Iron Chelators: New Opportunities and New Dilemmas," Haematologica, Vol. 91, No. 10, 2006, 1307-1312.

[16] G. Zanninelli, W. Breuer and Z. I. Cabancthik, "Daily Labile Plasma Iron as an Indicator of Chelator Activity in Thalassaemia Major Patients," British Journal Haematology, Vol. 147, No. 5, 2009, pp. 744-751. doi:10.1111/j.1365-2141.2009.07907.x

[17] A. Raptis, M. S. Duh, S. T. Wang, et al., "Treatment of Transfusional Iron Overload in Patients with Myelodysplastic Syndrome or Severe Anemia: Data from Multicenter Clinical Practices," Transfusion, Vol. 50, No. 1, 2010, pp. 190-199.

[18] H. A. Leitch, J. M. Chase, T. A. Goodman, et al., "Improved Survival in Red Blood Cell Transfusion Dependent Patients with Primary Myelofibrosis (PMF) Receiving Iron Chelation Therapy," Journal of Hematology Oncology, Vol. 28, No. 1, 2009, pp. 40-48.

[19] C. Rose, S. Brechignac, D. Vassilief, et al., "GFM (Groupe Francophone des Myélodysplasies).Does Iron Chelation Therapy Improve Survival in Regularly Transfused Lower Risk MDS Patients? A Multicenter Study by the GFM," Leukemia Research, Vol. 34, No. 7, 2010, pp. 864-870. doi:10.1016/j.leukres.2009.12.004

[20] P. D. Jensen, I. M. Jensen and J. Ellegaard, "Desferrioxamine Treatment Reduces Blood Transfusion Requirements in Patients with Myelodysplastic Syndrome," British Journal Haematology, Vol. 80, No. 1, 1992, pp. 121-124.

[21] E. Messa, D. Cilloni, F. Messa, et al., "Deferasirox Treatment Improved the Hemoglobin Level and Decreased Transfusion Requirements in Four Patients with the Myelodysplastic Syndrome and Primary Myelofibrosis," Acta Haematologia, Vol. 120, No. 2, 2008, pp. 70-74.

[22] C. Camaschella, A. Campanella, L. De Falco, et al., "The Human Counterpart of Zebrafish Shiraz Shows Sideroblastic-Like Microcytic Anemia and Iron Overload," Blood, Vol. 110, No. 4, 2007, pp. 1353-1358.

[23] V. Pullarkat, "Objectives of Iron Chelation Therapy in Myelodysplastic Syndromes: More than Meets the Eye?" Blood, Vol. 114, No. 26, 2009, pp. 5251-5255.

[24] J. Koreth, J. H. Antin, "Iron Overload in Hematologic Malignancies and Outcome of Allogeneic Hematopoietic Stem Cell Transplantation," Haematologica, Vol. 95, No. 3, 2010, pp. 364-366. 
[25] C. E. Cooper, G. R. Lynagh, K. P. Hoyes, et al., "The Relationship of Intracellular Iron Chelation to the Inhibition and Regeneration of Human Ribonucleotide Reductase," The Journal of Biological Chemistry, Vol. 271, No. 34, 1996, pp. 20291-20299. doi:10.1074/jbc.271.34.20291

Disclosure: C. H. received travel support and honoraria from Novartis Basel Switzerland and Apotex Toronto Canada. RPG is an employee of Celgene Corp, Summit, NJ.
[26] E. Angelucci, G M. Brittenham, C. E. McLaren, et al., "Hepatic Iron Concentration and Total Body Iron Stores in Thalassemia Major," The New England Journal Medicine, Vol. 343, No. 5, 2000, pp. 327-331. doi:10.1056/NEJM200008033430503 